\title{
Predictions of the mutual events of the Galilean Satellites of Jupiter occurring in 2002-2003
}

\author{
J.-E. Arlot` \\ Institut de mécanique céleste et de calcul des éphémérides - Observatoire de Paris, UMR 8028 du CNRS, \\ 77 avenue Denfert-Rochereau, 75014 Paris, France \\ Received 31 July 2001 / Accepted 21 November 2001

\begin{abstract}
Mutual occultations and eclipses of the Galilean satellites of Jupiter will occur in 2002-2003. This paper provides predictions of these events as well as information useful for their observations. Such events are uncommon, since they occur only every 6 years when the Earth and the Sun pass through the common orbital plane of the Galilean satellites. Therefore, we encourage professional and amateur astronomers to join the networks of observers in order to get as many observations as possible. Data on the predictions of the events are available on the web server of the IMCCE (www.bdl.fr).
\end{abstract}

Key words. planets and satellites: general - planets and satellites: individual: Jupiter

\section{Introduction}

The mutual events of the Galilean satellites have been extensively observed since 1973. Improvements in detectors have made the observations easier and studies have shown the importance of the observations: high astrometric precision of the relative positions of the involved satellites and information on the surfaces of the satellites themselves may be deduced from the observations. Having observations over long periods of time increases the possibility of obtaining results on the variations of surface aspects and also may allow the determination of the acceleration of Io and the other satellites. This encourages us to continue the observations and to predict these events.

\section{The mutual events}

\subsection{Occurrence of the events}

Since the Galilean satellites have their orbits almost in the same plane, mutual events occur regularly when the Earth (for the occultations) and the Sun (for the eclipses) pass through the orbital plane of the satellites. This plane corresponds closely to the equatorial plane of Jupiter. This occurrence takes place at the "equinox" on Jupiter, i.e. when the jovicentric declination of the Sun (and the Earth, which appears to be close to the Sun as seen from Jupiter) are near zero. Figure 1 shows the variation of these jovicentric declinations: the best periods

\footnotetext{
* e-mail: Jean-Eudes.Arlot@bdl.fr
}

for large, deep, mutual events appear to be January and June 2003 for the occultations and March 2003 for the eclipses. Fortunately, the opposition of Jupiter with the Sun occurs in February 2003, allowing observations from November 2002 to June 2003.

\subsection{Nature of the events}

The description of the mutual eclipses and occultations have been made in a previous paper, especially in technical notes made for the occurrence of the 1997 events, available on the web site of IMCCE at the address http://www.bdl.fr/phemu97_eng.html or in Arlot (1999).

The occultations consist of the arrival of the disk of the occulting satellite on that of the occulted satellite. Therefore, the amount of light received by a terrestrial observer decreases and increases during the event. The eclipses correspond to the arrival of the eclipsed satellite in the shadow cone of the eclipsing satellite. In that case, only the eclipsed satellite may be observed, leading, in some cases, to a total eclipse (the complete disappearance of the eclipsed satellite). During the occurrence of the mutual events, the magnitude of the events will be small at the beginning and the end of the period (the jovicentric declinations of the Earth and the Sun are not yet zero and the satellites are not exactly on the same line as seen from the Earth (or the Sun). Contrarily, the magnitude of the events will be larger for the zero value of the jovicentric declination of the Earth and the Sun. Observers 


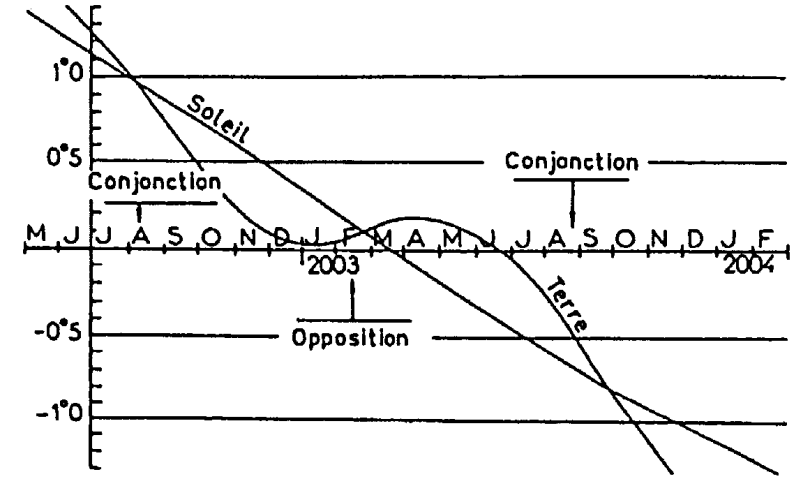

Fig. 1. Jovicentric declination of the Earth (Terre) and the Sun (Soleil).

should be aware that the signal/noise ratio will be larger for deep phenomena.

\section{The predictions for $2002-2003$}

\subsection{The model used for the predictions}

The prediction of the events requires the use of an accurate model of the motion of the Galilean satellites. In fact, the occurrence of the events is very sensitive to the small inclinations of the orbits of the Galilean satellites to the equatorial plane of Jupiter, which corresponds to the mean orbital plane of the Galilean satellites. Because of that, a difference of a few tenths of an arcsecond in the relative positions of the Galilean satellites may affect whether the event will occur or not. Therefore, we use one of the most accurate ephemerides based upon the theory of Sampson (1921), improved by Lieske (1977), and using the constants calculated by myself (Arlot 1982). This model, named G-5, is the one used for the ephemerides available on the web site of the IMCCE at the address: http://www.bdl.fr/ephem/ephesat/satformbis_eng.html.

The calculations for the prediction of the events differ for the occultations and for the eclipses. For the occultations, we determinate the time of the first contact, maximum and last contact of the two disks of the satellites as seen from the Earth. For the eclipses, we do not consider that they are occultations as seen from the Sun because of the light-time. Therefore, we calculate the first and last contact of the eclipsed satellite with the penumbral and umbral cones and the minimum distance to the axis of the cones. The relative positions of the cones and the satellites are calculated carefully taking into account the light-time. In some cases, the relative velocity of the two satellites or of the eclipsed satellite with reference to the cones is very small, or has non linear variations. This induces some errors in the times of the beginning and end of these events.

\subsection{The data}

The predicted mutual events are available with this paper in electronic form at the CDS via anonymous ftp to cdsarc.u-strasbg.fr (130.79.128.5) or via http://cdsweb.u-strasbg.fr/cgi-bin/qcat?J/A+A/383/719 and also on the web site of the IMCCE at the address: http://www.bdl.fr/ephem/ephesat/phenomena_eng.html. Table 1 provides the data detailed below and the printed Table 1 presents an example of the data for several days in February, 2003.

Columns 1, 2, 3: date of the maximum of the event.

Columns 4, 5, 6: nature of the event: 1 OCC 2 means that satellite 1 occults satellite 2, 3 ECL 4 means that satellite 3 eclipses satellite 4 , etc.

Column 7: $\mathrm{P}$ stands for partial, $\mathrm{A}$ for annular, $\mathrm{T}$ for total and blank means eclipse by the penumbra.

Columns 8, 9, 10: (only for eclipses): hour, min, s in UTC of the beginning of the eclipse by the penumbra.

Columns 11, 12, 13: hour, min, s in TT of the beginning of the eclipse by the shadow or beginning of the occultation.

Columns 14, 15, 16: (only for total events) hour, min, $\mathrm{s}$ in UTC of the beginning of totality.

Columns 17, 18, 19: hour, min, s in TT of the maximum of the event.

Columns 20, 21, 22: (only for total events) hour, min, $\mathrm{s}$ in UTC of the end of totality.

Columns 23, 24, 25: hour, min, s in TT of the end of the eclipse by the shadow or end of the occultation.

Columns 26, 27, 28: (only for eclipses): hour, min, s in TT of the end of the eclipse by the penumbra.

Columns 29: flux drop (if 0 , grazing event with a very small signal to be detected; if 1 , total event with a large signal easy to detect).

Column 30: duration of the event in seconds; no duration is indicated for grazing events.

Column 31: apparent distance from the occulted or eclipsed satellite to the center of Jupiter in jovian radii.

Column 32: impact parameter in arcsec.

The dates are provided in Terrestrial Time (TT) since the UTC for this period is not yet available. The difference TT - UTC will, however, be near $65 \mathrm{~s}$ in 2003 . Note that observations will be recorded referred to UTC.

Note that, in the list of events in Table 1, three events present two minima in the predicted light curves, because of the length of these events and of the changing velocity of the satellites during the events:

on December 20, 2002, J2 occults J1 with a first minimum distance at $21 \mathrm{~h} 40 \mathrm{~m}$ and a second one at $22 \mathrm{~h} 57 \mathrm{~m}$; also on June 22, 2003, J3 occults J1 with a first minimum at $9 \mathrm{~h} 45 \mathrm{~m}$ and a second one at $11 \mathrm{~h} 19 \mathrm{~m}$ and in 2003, August 25/26, J3 occults J2 from 20h 03m on Aug. 25 to $1 \mathrm{~h} 16 \mathrm{~m}$ on Aug. 26 with two minima, the first one at $20 \mathrm{~h} 54 \mathrm{~m}$ on June 25 and the second one at $0 \mathrm{~h} 18 \mathrm{~m}$ on Aug. 26.

Note also that the flux drop is calculated for uniform disks for any wavelength. The reduction of the observed light-curves should take into account the different parameters of reflectivity of the ground depending on the wavelengths used for the observations. 
Table 1. Mutual events occurring during the 2002-2003 period: example of data from the table available in electronic form at the CDS or at ftp://ftp.bdl.fr/pub/ephem/satel/phemu03/phemu03liste_eng.txt.

\begin{tabular}{|c|c|c|c|c|c|c|c|c|c|c|c|c|}
\hline col. 123 & 4567 & 8910 & 111213 & 141516 & 171819 & 202122 & 232425 & 262728 & 29 & 30 & 31 & 32 \\
\hline 2003220 & 1 ECL $2 \mathrm{P}$ & 162638 & 162744 & & 162852 & & 16300 & 16317 & .604 & 136 & .9 & .330 \\
\hline 2003220 & $1 \mathrm{OCC} 2 \mathrm{P}$ & & 154832 & & 155021 & & 15529 & & .381 & 217 & 1.3 & .183 \\
\hline 2003220 & 4 ECL $2 \mathrm{P}$ & 142358 & 142819 & & 142833 & & 142850 & 14338 & .543 & 32 & 2.3 & .462 \\
\hline 2003220 & 4 OCC $2 \mathrm{~T}$ & & 121625 & 121856 & 121934 & 122013 & 122244 & & .295 & 379 & 3.7 & .089 \\
\hline 2003222 & 2 OCC 1 & & & & 212422 & & & & .000 & 0 & 6.8 & 1.11 \\
\hline 2003222 & 2 ECL 1 & & & & 221512 & & & & .000 & 0 & 6.1 & 1.04 \\
\hline
\end{tabular}

During the 2002-2003 event season, 581 events have been predicted. Not all of them are observable: some will remain grazing with no detectable signal, some will occur behind Jupiter or during an eclipse by the planet and some will be difficult to observe because of the conjunction of Jupiter with the Sun. In the next subsections, we will provide more information on the visibility and on the observability of these events.

\subsection{The visibility of the events}

Since they occur anytime and since their duration is, usually, only a few minutes long, the events are observable only from selected sites where Jupiter is visible. The declination of Jupiter during the occurrence of the mutual events will be near +17 degrees. Therefore, the observations will be easier in the northern hemisphere. Table 2 provides the number of events observable from some selected sites around the world. We considered that an event is observable when the Sun is more than 10 degrees under the horizon and Jupiter is more than 15 degrees above the horizon. Table 3 provides the number of events depending on several criteria. Note that grazing events may be observable or not because of the sensitivity of these events to the inclinations of the orbital planes of the satellites. We considered that an event is grazing only when its observability depends on the possible error in the ephemerides. So, if a signal is detected during such an event, one will get valuable information. Note also that the total or annular events will present light curves with a flat step allowing the analysis of the scattering law of the light on the surfaces of the satellites. Short events or faint events (the magnitude of which being less than ten percent) will be difficult to catch because of the low signal to noise ratio, and observers will need near perfect photometric conditions. The long events (one hour or more) are interesting too, but the observers must take into account the changing elevation of Jupiter on the sky: the event must end before Jupiter sets. The observers may be aware that the predicted times may have an error of a few minutes in some cases, even if they are within a few seconds most of the time. This difference between the observation and the prediction contains information relevant to improving the theoretical models of the motions of the satellites. Therefore, the observations
Table 2. Visibility of the mutual events depending on the site of observation.

\begin{tabular}{lll}
\hline Observatory & $(1)$ & $(2)$ \\
\hline Alma-Ata (Kazakstan) & 150 & 49 \\
Nankin (China) & 148 & 50 \\
Mitaka (Japan) & 147 & 47 \\
Pic du Midi (France) & 140 & 43 \\
Kiev (Ukraine) & 138 & 41 \\
Kavalur (India) & 137 & 47 \\
Bucarest (Romania) & 137 & 47 \\
Paris (France) & 136 & 43 \\
Pico Veleta (Spain) & 136 & 41 \\
Canarian Islands (Spain) & 136 & 40 \\
Stuttgart (Germany) & 134 & 40 \\
Catania (Italy) & 134 & 40 \\
Haute-Provence (France) & 133 & 40 \\
Mc Donald (USA) & 132 & 41 \\
Uccle (Belgium) & 132 & 39 \\
Torino (Italy) & 132 & 38 \\
Barcelona (Spain) & 132 & 38 \\
Washington DC (USA) & 131 & 39 \\
Mauna Kea, Hawaii (USA) & 130 & 38 \\
Edmonton (Canada) & 129 & 41 \\
Topeka Kansas (USA) & 126 & 41 \\
Itajuba (Brazil) & 109 & 30 \\
Johannesburg (South Africa) & 106 & 34 \\
ESO (Chile) & 97 & 32 \\
Siding Spring (Australia) & 95 & 32 \\
\hline
\end{tabular}

(1): All events, even grazing.

(2): Events easily observable magnitude larger than 20 percent occurring far from Jupiter.

must start at least 10 min before the predicted beginning and must be referred to UTC within one tenth of a second.

Information on the visibility of the events from any site of observation is provided on the web site of IMCCE at the address given below. 
Table 3. Distribution of the events.

\begin{tabular}{|c|c|c|c|c|c|c|c|c|c|}
\hline & \multirow[t]{2}{*}{ Dates } & \multicolumn{2}{|c|}{ number } & \multicolumn{2}{|c|}{ of } & \multicolumn{4}{|l|}{ events } \\
\hline & & (1) & $(2)$ & $(3)$ & (4) & $(5)$ & $(6)$ & $(7)$ & $(8)$ \\
\hline \multirow[t]{3}{*}{2002} & October & 9 & 1 & 1 & 0 & 3 & 0 & 9 & 67 \\
\hline & November & 28 & 2 & 0 & 7 & 7 & 7 & 21 & 93 \\
\hline & December & 64 & 5 & 14 & 10 & 32 & 10 & 54 & 123 \\
\hline \multirow[t]{9}{*}{2003} & January & 89 & 6 & 8 & 29 & 39 & 27 & 62 & 157 \\
\hline & February & 56 & 4 & 18 & 8 & 36 & 24 & 32 & 169 \\
\hline & March & 68 & 1 & 15 & 24 & 39 & 49 & 19 & 136 \\
\hline & April & 63 & 2 & 9 & 22 & 32 & 45 & 18 & 105 \\
\hline & May & 52 & 3 & 10 & 10 & 33 & 26 & 26 & 78 \\
\hline & June & 65 & 3 & 5 & 15 & 23 & 21 & 44 & 54 \\
\hline & July & 35 & 6 & 10 & 10 & 17 & 4 & 31 & 31 \\
\hline & August & 47 & 0 & 8 & 28 & 27 & 22 & 25 & 9 \\
\hline & September & 13 & 1 & 0 & 3 & 5 & 8 & 5 & 14 \\
\hline
\end{tabular}

(1): Total number of events; (2): number of grazing events; (3): number of total or annular events; (4): number of events the duration of which is more than $20 \mathrm{~s}$ and less than $1200 \mathrm{~s}$; (5): number of partial events the magnitude of which is more than 20 percent; (6): number of eclipses; (7): number of occultations; (8): mean distance Sun-Jupiter in degrees.

\section{The observation of the mutual events}

\subsection{The receptors}

The observation of the mutual events consists of the recording of the light flux as a function of time using a photometric receptor. At the present time, CCDs are the most common detectors used for this type of observation:

- the speed of the acquisition of the images allows the recording of at least 3 images per second (for short events);

- each image can be dated in UTC within an accuracy of $0.1 \mathrm{~s}$ in order to get an astrometric accuracy of 1 kilometer for the relative position of the satellites.

Video cameras may also be used, thanks to the brightness of the satellites. However, be sure to record the UTC time on each image. The advantage of two-dimensional receptors is the possibility to get several satellites in the field. This will help for the photometric reduction, as we will see in the next paragraph, "Calibration".

Other types of detectors may be used, such as single channel photoelectric photometers for fast photometry in several spectral bands.

Photographic or visual observations should be avoided because of the poor photometric accuracy. In fact, only long events may be recorded using the photographic technique. Visual observations of deep events not longer than 10 min should be made only in cases of failure of electronic receptors!

Technical notes on the use of several types of detectors are available by request from the author or on the web server of IMCCE at the address given below.

\subsection{The calibration}

Most of time, the raw light-curves are difficult to analyse without a specific photometric reduction. Since we need only relative photometry (the recorded magnitude drop is measured referred to the magnitude of the satellites before and after the event). However, it is important to record a reference object not affected by the event at the same time. Using a two-dimensional receptor will allow the observer to simultaneously record the satellites involved in the event, and another satellite not involved, with constant brightness. Only for long events will it be useful to take into account the rotation of the satellites. A twodimensional detector will also record the sky background simultaneously. Then, the reduction will easily solve the problems related to light clouds or to the variation of the elevation of the observed bodies in the sky, as well as to the brightness of the sky background before sunrise or after sunset. If using a single channel receptor, the observer will need to alternatively record the sky background and a reference object either during the event for a long event (more than $20 \mathrm{~min}$ ) or just before and after the event for a short event.

\subsection{The PHEMU03 campaign of observations}

In order to optimize the observations and to catch the maximum number of phenomena, we propose to organize a campaign of observations for the coordination of the observing sites. Such campaigns, organized in the past, allowed publication of a very complete catalogue of data such as in Arlot et al. (1997) for the 1991 occurrence and in Arlot et al. (2001, in preparation) and Emelianov et al. (2000) for the 1997 occurrence. The reader is 
encouraged to join our campaign of observations, to contact the author at arlot@bdl.fr and to get information on the campaign of observations from the web address www.bdl.fr/phemu03_eng.html.

\section{The interest of the observations}

\subsection{Astrometry of the Galilean Satellites}

The goal of the observation of the mutual phenomena of the Galilean Satellites of Jupiter is to obtain astrometric data leading to relative positions of the satellites with high accuracy. Observations of the mutual events have been shown to be more accurate than photographic or CCD ones. The measurement of the magnitude drop at the time of the minimum distance corresponds to the measurement of this minimum distance. Thanks to the absence of an atmosphere on the satellites, the observations are more interesting than thoses of the eclipses by Jupiter because of the sharpness of the light curves. Eclipse observations lead to relative positions determined within an accuracy of $1000 \mathrm{~km}$, photographic or CCD positions within $500 \mathrm{~km}$ and mutual events within $200 \mathrm{~km}$. This accuracy for the mutual events may be improved to $30 \mathrm{~km}$ using a more sophisticated reduction involving surface reflectivity.

Such observed positions help to fit the dynamical models of the motions of the satellites. Their accuracy allows a better determination of the eccentricities which should constrain the model of tidal effects on Io and Europe and the model of the interior of these satellites. Also, these observations allow detection of non-gravitational forces, especially for Io, through the determination of an acceleration. Space probes have made some precise observations during a too short interval of time to be able to determine an acceleration. For that, we need accurate observations over a long interval of time as provided by the mutual events. The first modern observations of these events started in 1973, providing us with thirty years of data necessary in order to measure an acceleration in the motion of the satellites.

\subsection{Planetology: Analysis of the surfaces}

Looking for a better reduction of the mutual phenomena, Vasundhara et al. (1996) demonstrated that a new reduction considering that the satellites are not uniform disks may help to improve the accuracy of the data. Surface effects were suspected to be non-negligible when observing the mutual events presenting light curves with an asymmetrical aspect. Taking into account the surface effects for the reduction allows the determination of unknowns related to to planetologic parameters such as the porosity and the rugosity of the surfaces of the satellites themselves, implying different scattering laws affecting the observed light curves. Moreover, the observations in infrared wavelengths allow detection of volcanos and hot spots on the surface of the satellite Io, and measurement of their activity at the time of the observation (Descamps et al. 1992). A further publication will provide the dates of occultations of the main hotspots on Io.

\section{Conclusion}

The occurrence of the mutual events in 2002-2003 is particularly favorable because of the opposition of Jupiter when the mutual events are the most numerous (i.e., when the jovicentric declination of the Sun and the Earth is at a minimum). Furthermore, the declination of Jupiter itself at the date of the events will be around +17 degrees, very favorable for observation from most of the observatories around the world.

We encourage observers to make the most of observations during this occurrence in order to complete the collections of data gathered since 1973. Thirty years of such accurate data will allow a major improvement in the ephemerides of the Galilean Satellites.

Acknowledgements. These calculations have been made possible thanks to the CNRS (Centre National de la Recherche Scientifique) and the Institut de mécanique céleste/ Observatoire de Paris.

I also wish to thank Nicolas Fauvel (EPF) for his help in the calculation of the events.

\section{References}

Arlot, J.-E. 1982, A\&A, 107, 305

Arlot, J.-E., Ruatti, C., Thuillot, W., et al. 1997, A\&AS, 125, 399

Arlot, J.-E. 1999, Proc. of the 5th workshop PHEMU97, held in Catania, Italy on March 4-6, 1997, ed. J.-E. Arlot, \& C. Blanco (IMC Editions, Paris, ISBN 2-910015-25-4), 59, 75

Descamps, P., Arlot, J.-E., Thuillot, W., et al. 1992, Observations of the volcanoes of Io, Loki and Pele, made in 1991 at the ESO during an occultation by Europa, Icarus 100,235

Emelianov, N. V., A.A. Berejnoi, S. N., Vashkovjak, E. A., et al. 2000, A\&AS, 141, 433

Lieske, J. H. 1977, A\&A, 56, 333

Sampson, R. A. 1921, Memoirs of the Royal Astron. Soc., 63, 1

Vasundhara, R., Arlot, J.-E., \& Descamps, P. 1996, Proc. of the 172 IAU Symp., ed. S. Ferraz Mello, B. Morando, \& J.-E. Arlot (Kluwer Acad. Pub.), 423 